\title{
An Improved Synthesis of Pyran-3,5-dione, Application to the Synthesis of ABT-598, A Potassium Channel Opener, via Hantzsch Reaction
}

\author{
Wenke Li, * Gregory S. Wayne, John E. Lallaman, and Steven J. Wittenberger \\ Process Chemistry, Global Pharmaceutical Research and Development, Abbott Laboratories, \\ North Chicago, Illinois 60064-6285, U.S.A. \\ wenke.li@abbott.com
}

SUPPORTING INFORMATION

Table of Contents

\section{Experimental Procedures}

General.

4-Fluoro-3-iodobenzaldehyde (5).

Triethylamine salt of biscondensation product (6).

ABT-598,

${ }^{1} \mathrm{H}$ - and ${ }^{13} \mathrm{C}$-NMR Spectra

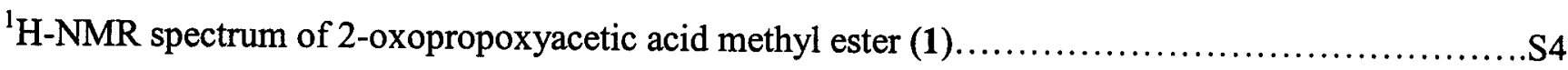

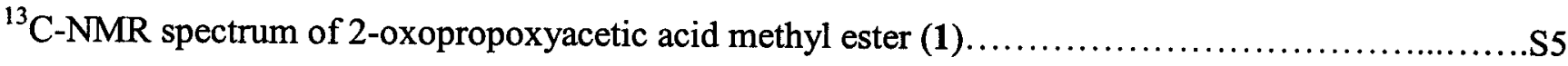

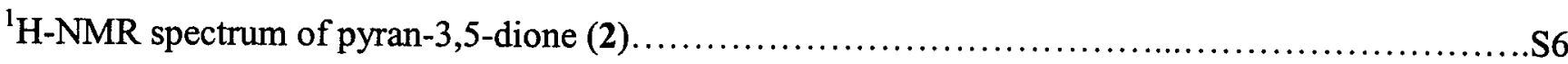

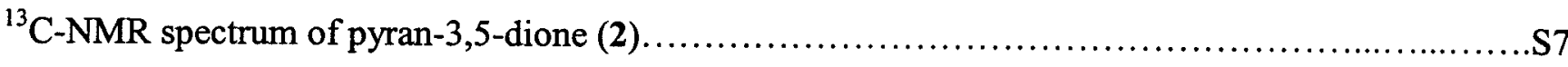

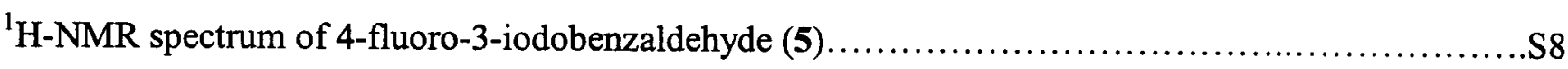

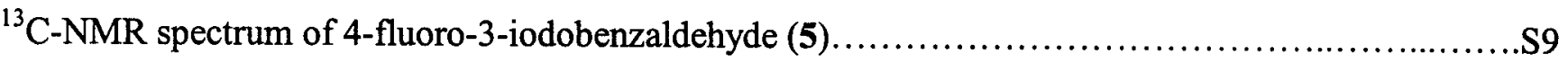

${ }^{1} \mathrm{H}$-NMR spectrum of triethylamine salt of biscondensation product (6) ............................ 10

${ }^{13} \mathrm{C}$-NMR spectrum of triethylamine salt of biscondensation product (6)............................. 11

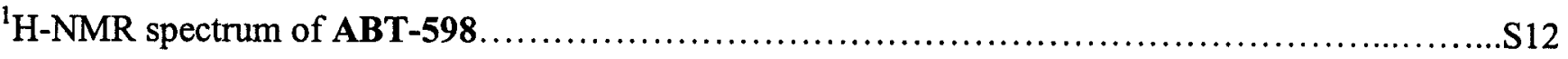

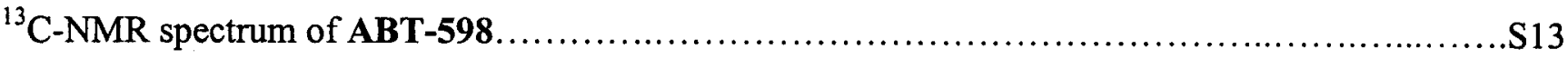




\section{General}

The NMR spectra were recorded on 400 or $600 \mathrm{MHz}$ instruments. All the reactions were performed under nitrogen. All chemicals and reagents were purchased and used without further purification unless otherwise mentioned. All melting points were uncorrected. HPLC purity was given as area normalization at $230 \mathrm{~nm}$. All assayed yields were obtained by HPLC against purified reference standards.

4-Fluoro-3-iodobenzaldehyde (5) ${ }^{1}$ To a solution of 4-fluorobenzaldehyde $(1 \mathrm{~kg}, 8.06 \mathrm{~mol})$, acetic acid (2 L), and $N$-iodosuccinimide $(2.17 \mathrm{~kg}, 9.64 \mathrm{~mol})$ was slowly added concentrated sulfuric acid (2 L). The resulting mixture was stirred at $40^{\circ} \mathrm{C}$ until HPLC analysis showed that di-iodo $5^{\prime}$ was $>1.5$ area $\%$. The reaction mixture was cooled to $14^{\circ} \mathrm{C}$ and the product precipitated by adding distilled water $(10 \mathrm{~L})$. The product was filtered and the wetcake washed with distilled water $(2 \mathrm{~L})$. The wetcake was redissolved in ethyl acetate/heptane $(11 \mathrm{~L} ; 1: 1 \mathrm{v} / \mathrm{v})$ containing approximately $100 \mathrm{ppm} \mathrm{BHT}^{2}$ After $^{2}$ stirring for $60 \mathrm{~min}$, the solution was filtered and the filtrate washed with $10 \%$ aqueous sodium thiosulfate $(5 \mathrm{~kg})$, then with $10 \%$ aqueous sodium carbonate $(5 \mathrm{~kg})$ and finally with distilled water $(5 \mathrm{~L})$. Analysis of the organic solution by HPLC indicated the presence of $1.42 \mathrm{~kg} 5(70 \%)$. The product was recrystallized from heptane twice to afford $1.07 \mathrm{~kg}$ (53\%) of 5 with < 0.4\% pa of 5'. mp: $67-67{ }^{\circ} \mathrm{C}$. ${ }^{1} \mathrm{H}$ NMR (400 MHz/CDCl $\left.{ }_{3}\right) \delta 9.91(\mathrm{~s}, 1 \mathrm{H}), 8.31(\mathrm{dd}, J=2.1,6.0 \mathrm{~Hz}, 1 \mathrm{H}), 7.88(\mathrm{ddd}, J=2.1,4.9,8.5 \mathrm{~Hz}$, $1 \mathrm{H}), 7.22(\mathrm{t}, J=8.1 \mathrm{~Hz}, 1 \mathrm{H}) .{ }^{13} \mathrm{C} \mathrm{NMR}\left(100 \mathrm{MHz} / \mathrm{CDCl}_{3}\right) \delta 188.7,165.1\left(\mathrm{~d}, J_{\mathrm{F}}=254 \mathrm{~Hz}\right), 141.2\left(\mathrm{~d}, J_{\mathrm{F}}\right.$ $=3.8 \mathrm{~Hz}), 134.2\left(\mathrm{~d}, J_{\mathrm{F}}=3.0 \mathrm{~Hz}\right), 131.7\left(\mathrm{~d}, J_{\mathrm{F}}=9.4 \mathrm{~Hz}\right), 116.4\left(\mathrm{~d}, J_{\mathrm{F}}=24.7 \mathrm{~Hz}\right), 82.4\left(\mathrm{~d}, J_{\mathrm{F}}=26.9 \mathrm{~Hz}\right)$. IR (neat) $2854,2757,1697,1688,1583,1483,1367,1240,1196 \mathrm{~cm}^{-1}$. Anal. Calcd for $\mathrm{C}_{7} \mathrm{H}_{4} \mathrm{FIO}: \mathrm{C}$, 33.63; H, 1.61. Found: C, 33.71; H, 1.50 .

Triethylamine salt of biscondensation product (6) The crude diketone sodium salt $2 . \mathrm{Na}(1.09 \mathrm{~kg}$, $8.0 \mathrm{~mol}$ ) was dissolved in water $(4.8 \mathrm{~L})$ and the $\mathrm{pH}$ of the resulting solution adjusted to 1.2 with $6 \mathrm{M}$ hydrochloric acid $(2.16 \mathrm{~kg})$. The acidic aqueous solution was extracted three times with ethyl acetate $(15.5 \mathrm{~L}$ each) and the extracts were concentrated. The residue was dissolved in ethyl acetate $(18 \mathrm{~L})$ at 45 ${ }^{\circ} \mathrm{C}$ and the solution was filtered to remove some insoluble material. The residual water level in filtrate was reduced by azetropic distillation with ethyl acetate several times. To the resulting yellow slurry was added 4-fluoro-3-iodobenzaldehyde (989.0 g, $3.96 \mathrm{~mol})$, isopropanol $(3.5 \mathrm{~L})$ and triethylamine $(599 \mathrm{~g}$, $5.9 \mathrm{~mol})$. The resulting solution was heated at $50^{\circ} \mathrm{C}$ until $<2 \% 2$ remained by HPLC analysis $(\sim 1 \mathrm{~h})$. The resulting slurry was cooled to $25^{\circ} \mathrm{C}$ and stirred at this temperature for $14 \mathrm{~h}$, then at $0^{\circ} \mathrm{C}$ for $1.5 \mathrm{~h}$. The slurry was filtered and the wetcake washed with isopropanol $(3.5 \mathrm{~L})$. The product was dried under 
vacuum with nitrogen bleed at $55^{\circ} \mathrm{C}$ to afford $1.96 \mathrm{~kg}$ of 6 (100\% potency; $88 \%$ yield). mp: $167-168$ ${ }^{\circ} \mathrm{C} . \quad{ }^{1} \mathrm{H}$ NMR $\left(400 \mathrm{MHz} / \mathrm{CDCl}_{3}\right) \delta 15.9$ (s, br, $\left.1 \mathrm{H}\right), 7.47-7.50(\mathrm{~m}, 1 \mathrm{H}), 7.10-7.16(\mathrm{~m}, 1 \mathrm{H}), 6.87(\mathrm{t}, J=$ $8.23, \mathrm{~Hz}, 1 \mathrm{H}), 5.88(\mathrm{~m}, 1 \mathrm{H}), 4.17(\mathrm{~s}, \mathrm{br}, 8 \mathrm{H}), 3.21(\mathrm{q}, J=7.27 \mathrm{~Hz}, 6 \mathrm{H}), 1.25(\mathrm{t}, J=7.27 \mathrm{~Hz}, 9 \mathrm{H}) .{ }^{13} \mathrm{C}$ NMR (150 MHz/CDCl 3 ) $\delta 192.3$ (br), 182.4 (br), 160.3, 158.7, 140.0, 137.5, 128.6, 114.7, 113.4, 80.7, 70.2 (br), 46.4, 27.8, 8.7. IR (neat) 3063, 2888, 1634, 1577, 1483, 1401, 1144. Anal. Calcd for $\mathrm{C}_{23} \mathrm{H}_{29} \mathrm{FINO}_{6}: \mathrm{C}, 49.21 ; \mathrm{H}, 5.21 ; \mathrm{N}, 2.50$. Found: $\mathrm{C}, 49.16 ; \mathrm{H}, 4.99 ; \mathrm{N}, 2.36$.

ABT-598 The mixture of $6(1.90 \mathrm{~kg}, 3.38 \mathrm{~mol})$, acetic acid $(9.97 \mathrm{~kg})$, distilled water $(190 \mathrm{~g})$ and ammonium acetate $(1.30 \mathrm{~kg}, 16.9 \mathrm{~mol})$ was heated to $105^{\circ} \mathrm{C}$ and stirred until $<1 \% 6$ remained by HPLC analysis $(\sim 1 \mathrm{~h})$. The reaction mixture was then cooled to $25^{\circ} \mathrm{C}$ and filtered. The wetcake was washed with ethanol $(9.4 \mathrm{~L})$ and air-dried to give $1.33 \mathrm{~kg}$ crude product. This was then dissolved in a solution of ethanol $(21.5 \mathrm{~L})$, distilled water $(2.3 \mathrm{~L})$ and potassium hydroxide $(238 \mathrm{~g})$. This crude product solution was filtered through an in-line filter into a $50 \mathrm{~L}$ flask, and cooled to $10^{\circ} \mathrm{C}$. A solution of $0.4 \mathrm{M}$ $\mathrm{HCl}$ was then added slowly until the $\mathrm{pH}$ reached 6.7 , while maintaining the temperature below $20^{\circ} \mathrm{C}$. The yellow slurry was filtered and the wetcake washed with $1: 1$ ethanol/water $(5.4 \mathrm{~L})$ and then with ethanol $(3.3 \mathrm{~L})$. After drying at $65^{\circ} \mathrm{C}$ overnight under vacuum with nitrogen bleed, $1.12 \mathrm{~kg}$ of ABT-598 was obtained (75\% yield from 6). ${ }^{1} \mathrm{H}$ NMR (400 MHz/DMSO) $\delta 10.07$ (br s, 1H), 7.57 (dd, $J=2.2,6.2$ $\mathrm{Hz}, 1 \mathrm{H}$ ), 7.20 (ddd, $J=2.2,5.1,8.5 \mathrm{~Hz}, 1 \mathrm{H}), 7.13(\mathrm{t}, J=8.2 \mathrm{~Hz}, 1 \mathrm{H}), 4.90(\mathrm{~s}, 1 \mathrm{H}), 4.43-4.55(\mathrm{~m}, 4 \mathrm{H})$, $4.04(\mathrm{~s}, 4 \mathrm{H}) .{ }^{13} \mathrm{C}$ NMR (100 MHz/DMSO) $\delta 190.6,158.9\left(\mathrm{~d}, J_{\mathrm{F}}=239 \mathrm{~Hz}\right), 149.3,143.2\left(\mathrm{~d}, J_{\mathrm{F}}=3 \mathrm{~Hz}\right)$, $137.2,129.0\left(\mathrm{~d}, J_{\mathrm{F}}=7 \mathrm{~Hz}\right), 115.0\left(\mathrm{~d}, J_{\mathrm{F}}=24 \mathrm{~Hz}\right), 108.9,81.6\left(\mathrm{~d}, J_{\mathrm{F}}=26 \mathrm{~Hz}\right), 71.1,63.2,29.9$. IR (neat) $3213,1657,1647,1634,1618,1485,1357,1236,1120,1121$. HRMS: calcd for $\mathrm{C}_{17} \mathrm{H}_{14} \mathrm{FINO}_{4}\left(\mathrm{M}^{\mathrm{N}}\right.$ $+\mathrm{H})$ : 441.9946. Found: 441.9944.

Reference and Note

1. The compound was previously made by a different method. Carroll, W. A., Altenbach, R. J., Bai, H., Brioni, J. D., Brune, M. E., Buckner, S. A., Cassidy, C., Chen, Y., Coghlan, M. J., Daza, A. V., et al. J. Med. Chem. 2004, 47, 3163-3179.

2. 4-Fluoro-3-iodobenzaldehyde is very susceptible to air-oxidation. However, adding $100 \mathrm{ppm}$ of butylated hydroxytoluene (BHT) to the recrystallization solvent can completely inhibit this degradation. 


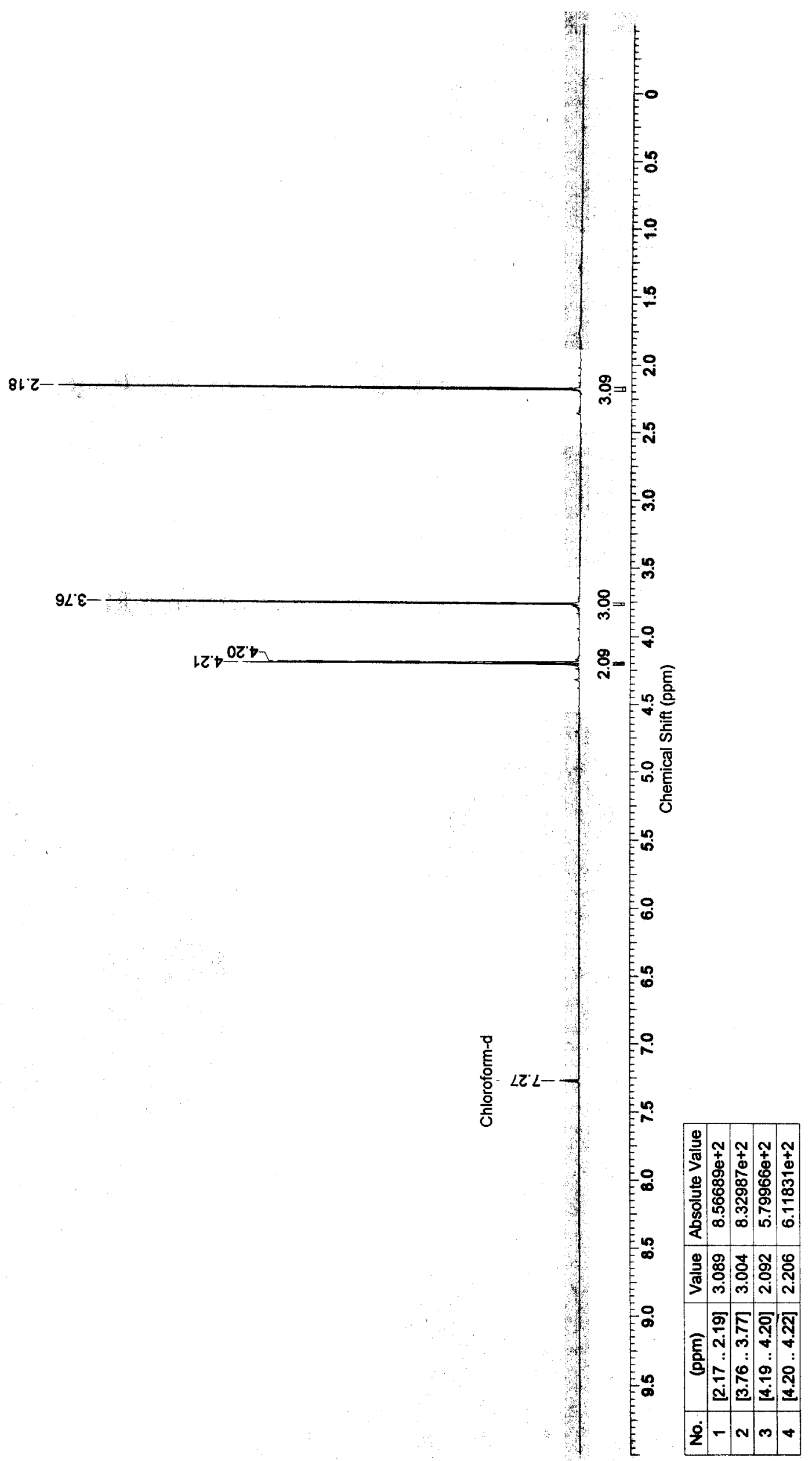




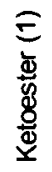

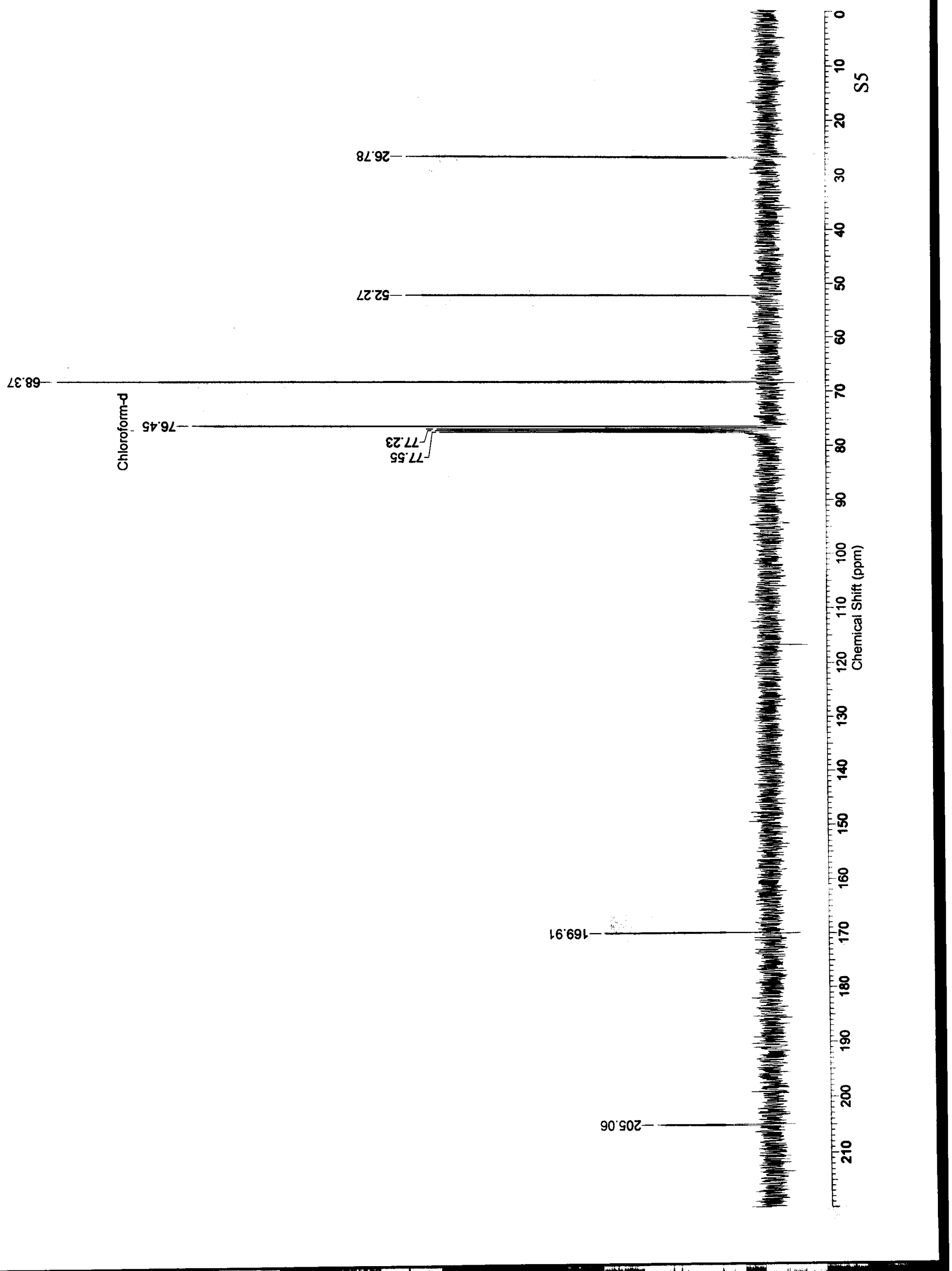




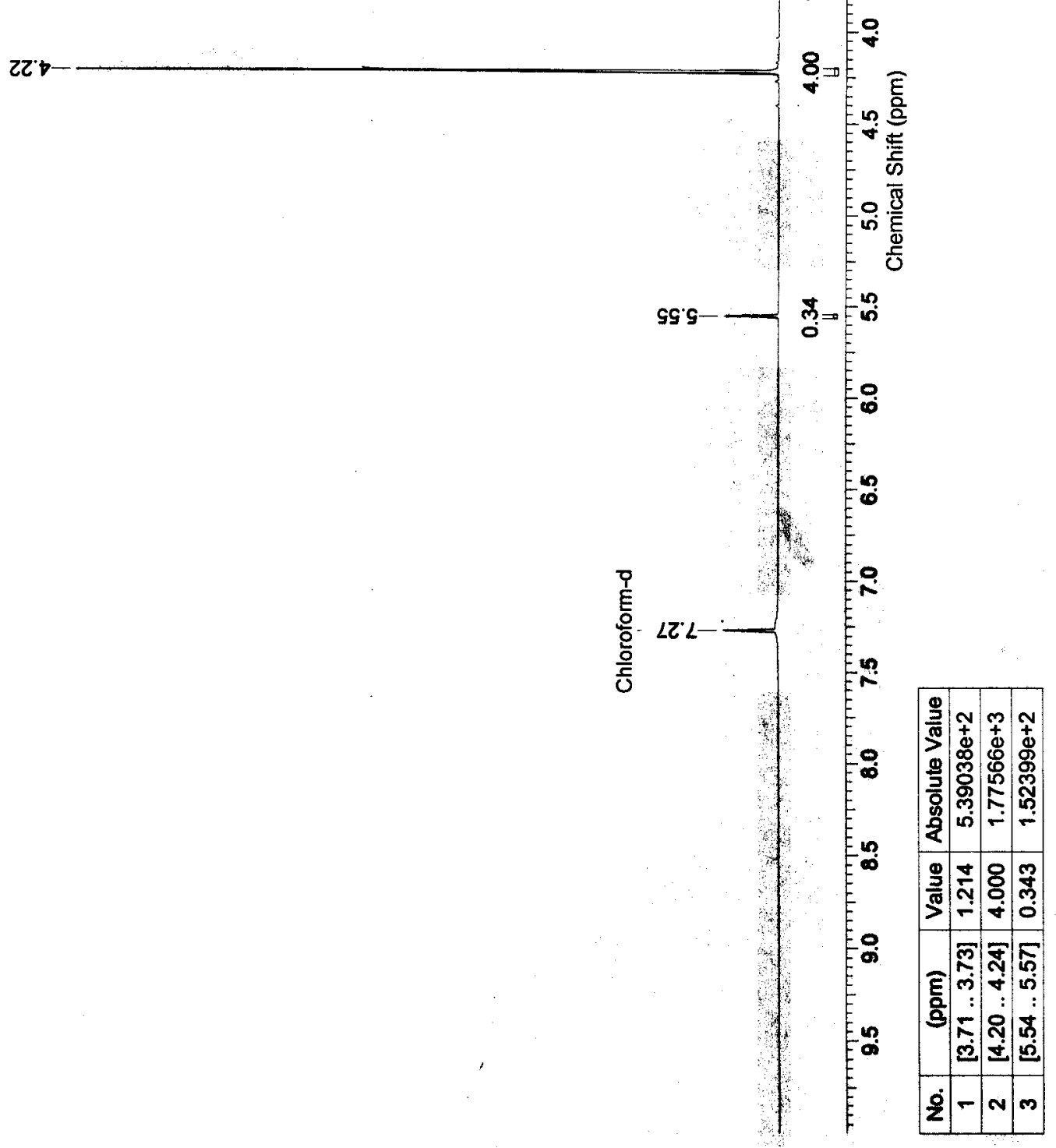




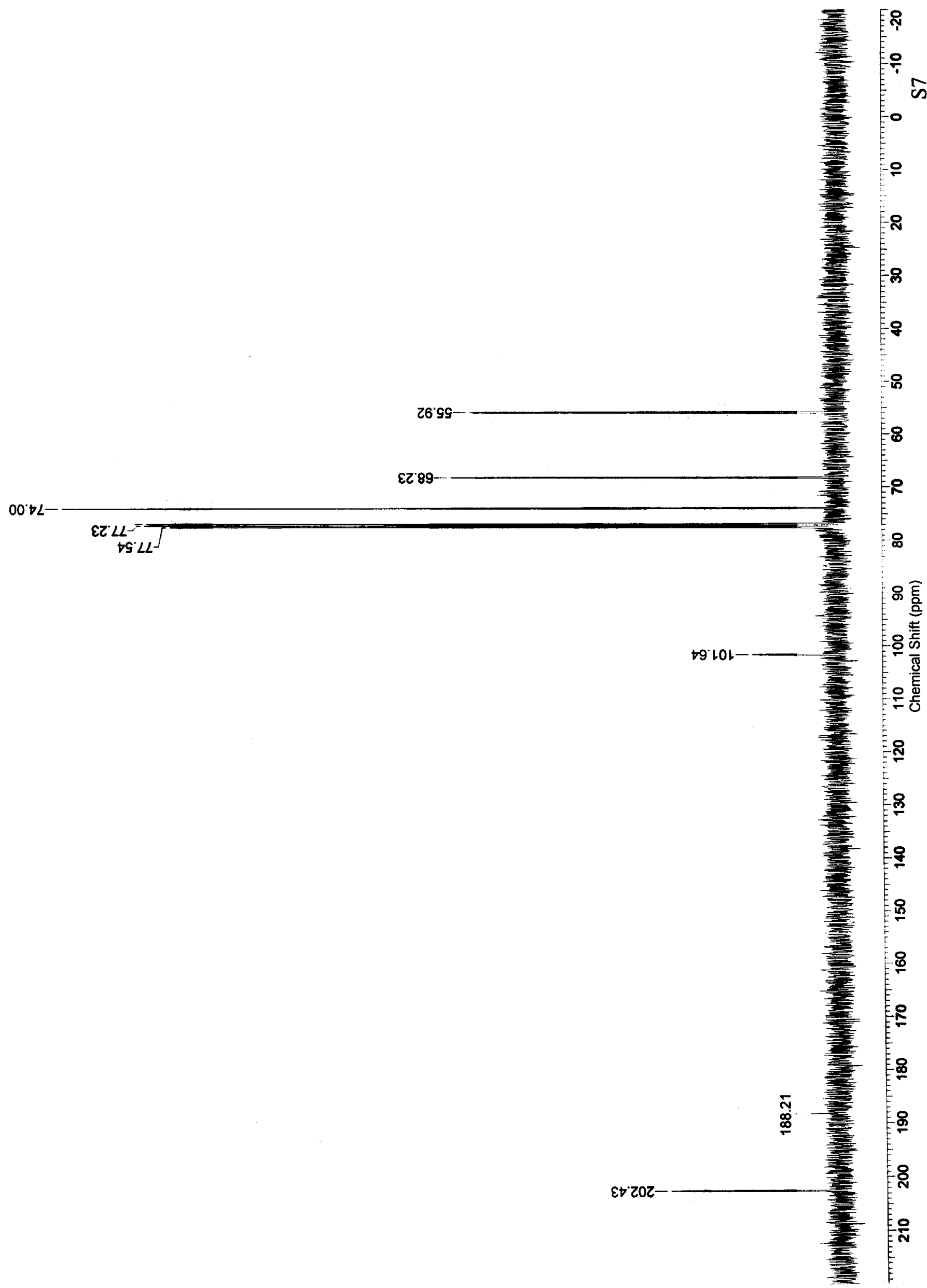




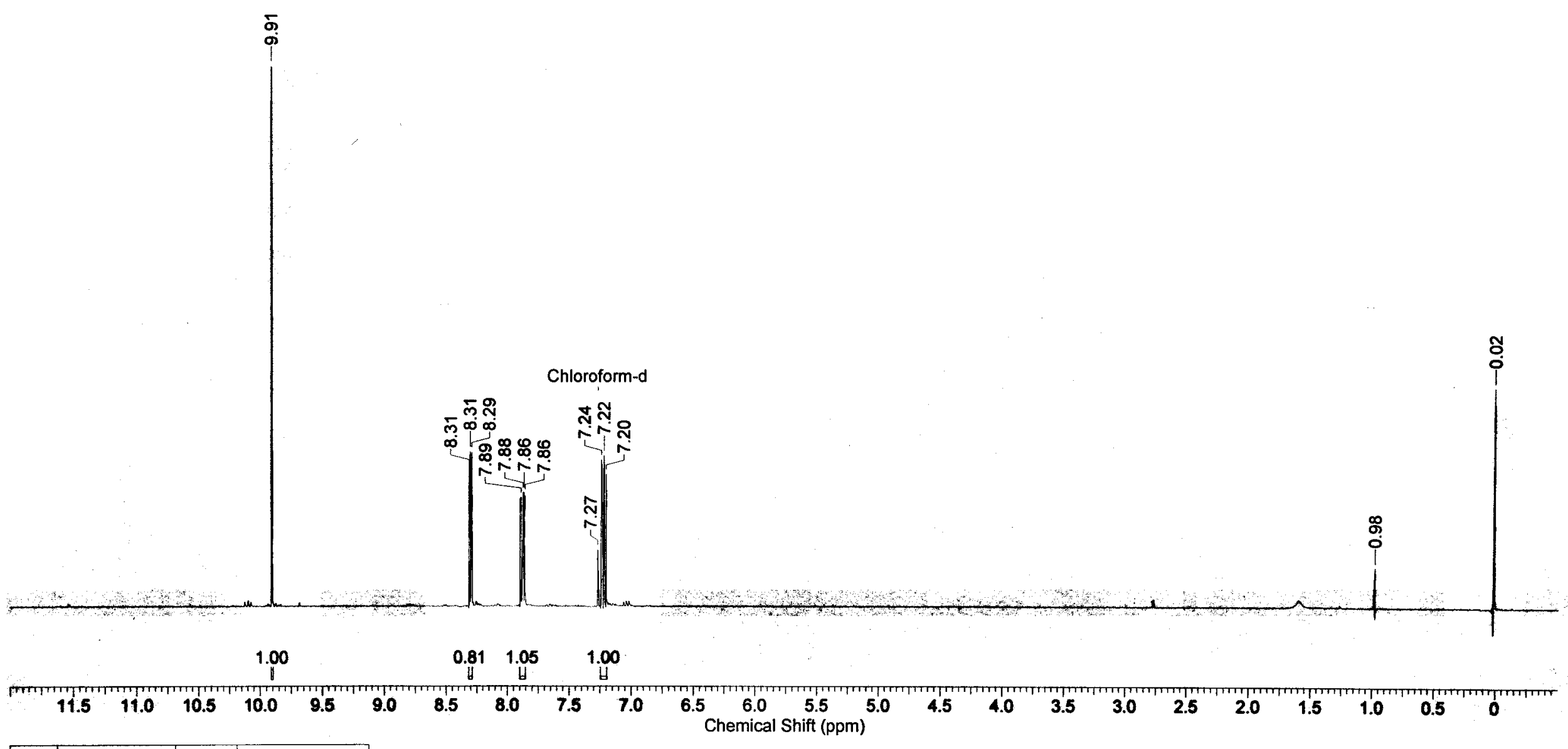

\begin{tabular}{|c|c|c|c|}
\hline No. & (ppm) & Value & Absolute Value \\
\hline 1 & {$[7.20 \ldots 7.25]$} & 1.000 & $1.19457 \mathrm{e}+3$ \\
\hline 2 & {$[7.86 . .7 .90]$} & 1.052 & $1.25674 \mathrm{e}+3$ \\
\hline 3 & {$[8.29 . .8 .32]$} & 0.811 & $9.68750 \mathrm{e}+2$ \\
\hline 4 & {$[9.90 \ldots 9.91]$} & 0.997 & $1.19056 \mathrm{e}+3$ \\
\hline
\end{tabular}


16.94

$\varepsilon Z \angle L-$ $\rightarrow S^{\prime} L L^{\prime}$

$\llcorner\varepsilon .9 เ$

99. 9 .
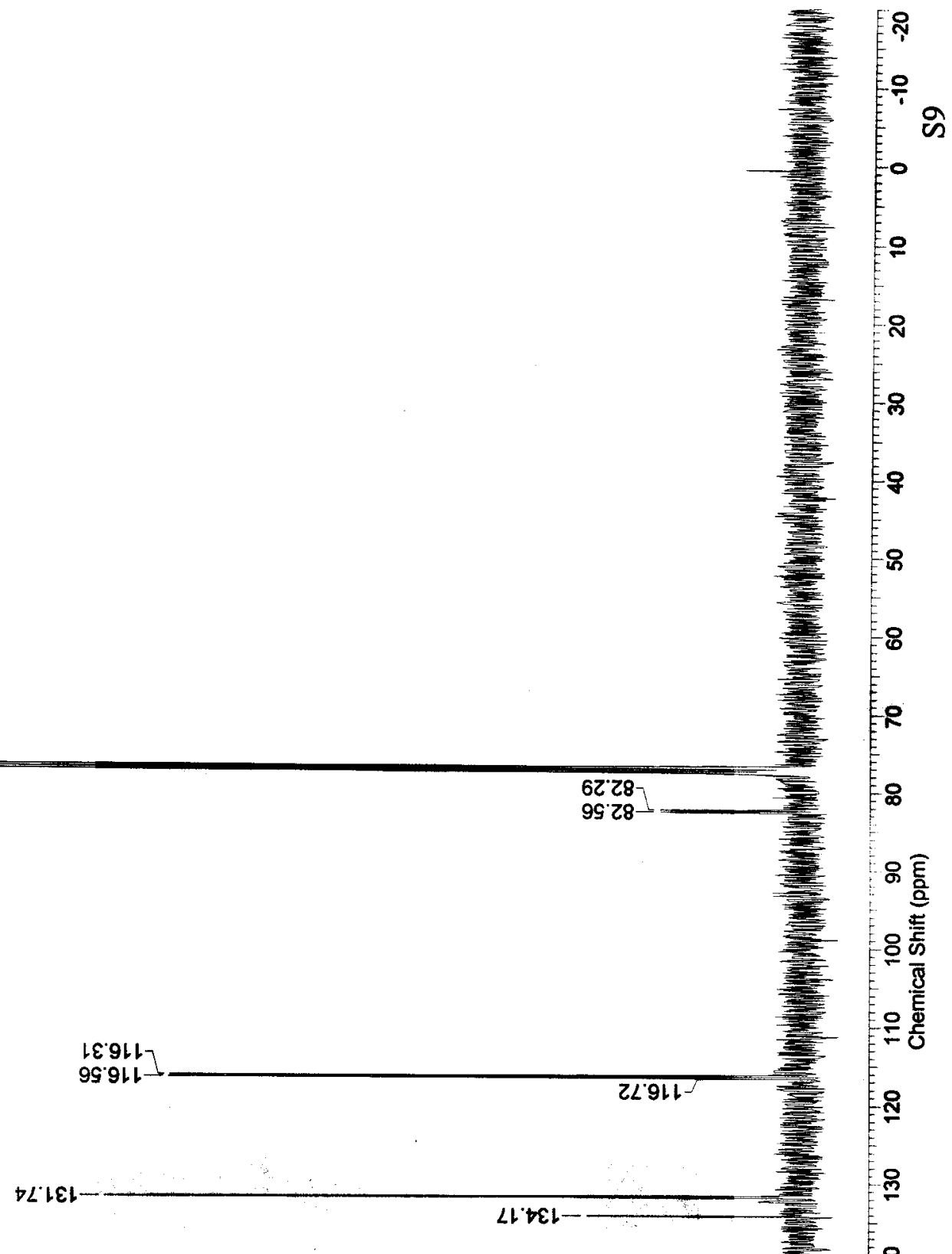

$81 \cdot L b$

† '

$9 \varepsilon \cdot 991$

7 


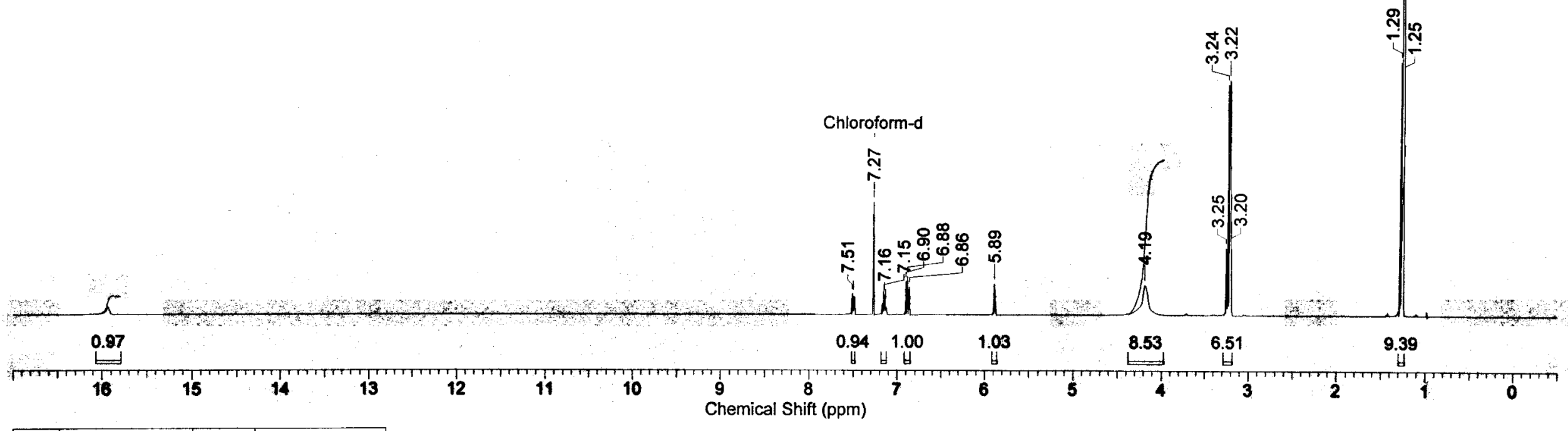

\begin{tabular}{|c|c|c|c|}
\hline No. & (ppm) & Value & Absolute Value \\
\hline 1 & {$[1.23 . .1 .30]$} & 9.394 & $2.44619 \mathrm{e}+3$ \\
\hline 2 & {$[3.18 . .3 .29]$} & 6.507 & $1.69437 \mathrm{e}+3$ \\
\hline 3 & {$[3.97 . .4 .38]$} & 8.528 & $2.22078 \mathrm{e}+3$ \\
\hline 4 & {$[5.86 . .5 .92]$} & 1.025 & $2.67019 \mathrm{e}+2$ \\
\hline 5 & {$[6.85 . .6 .92]$} & 1.000 & $2.60353 \mathrm{e}+2$ \\
\hline 6 & {$[7.12 . .7 .18]$} & 1.006 & $2.61875 \mathrm{e}+2$ \\
\hline 7 & {$[7.48 . .7 .52]$} & 0.941 & $2.45123 \mathrm{e}+2$ \\
\hline 8 & {$[15.79 . .16 .07]$} & 0.973 & $2.53346 \mathrm{e}+2$ \\
\hline
\end{tabular}




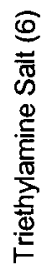

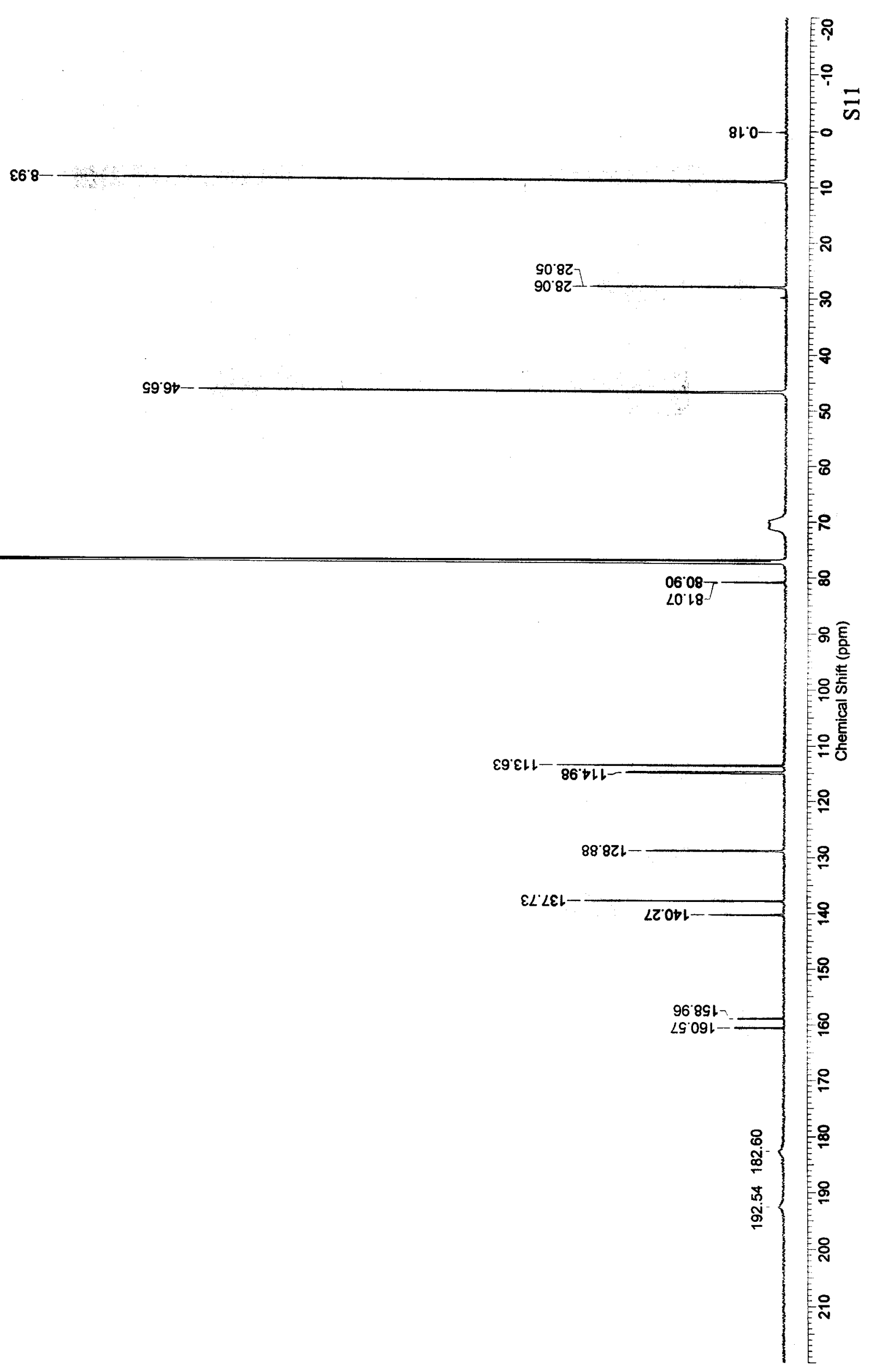


$\$ 0$

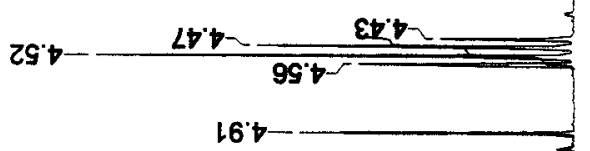

$\angle 001$

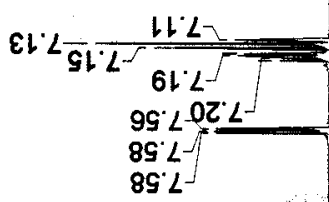

$09^{2} 2-$

$\varepsilon \varepsilon \varepsilon$

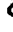

总

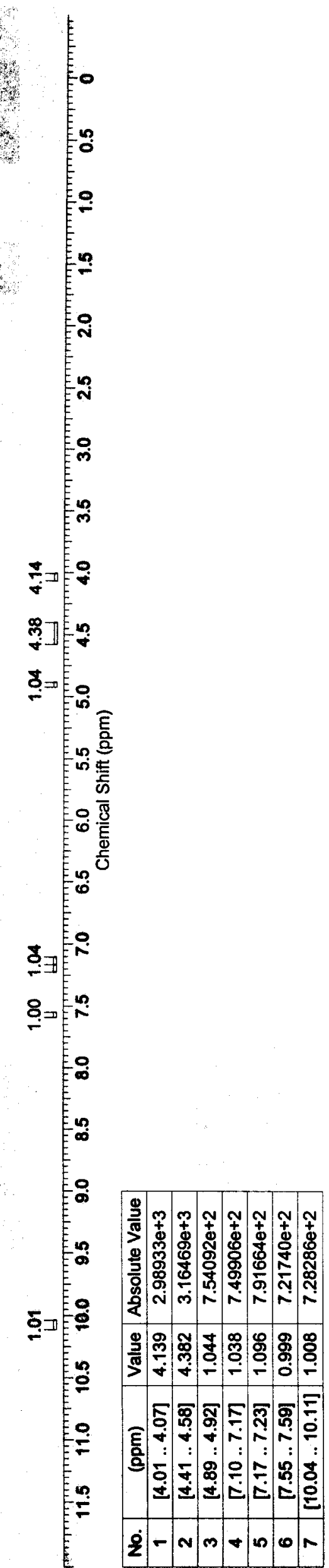




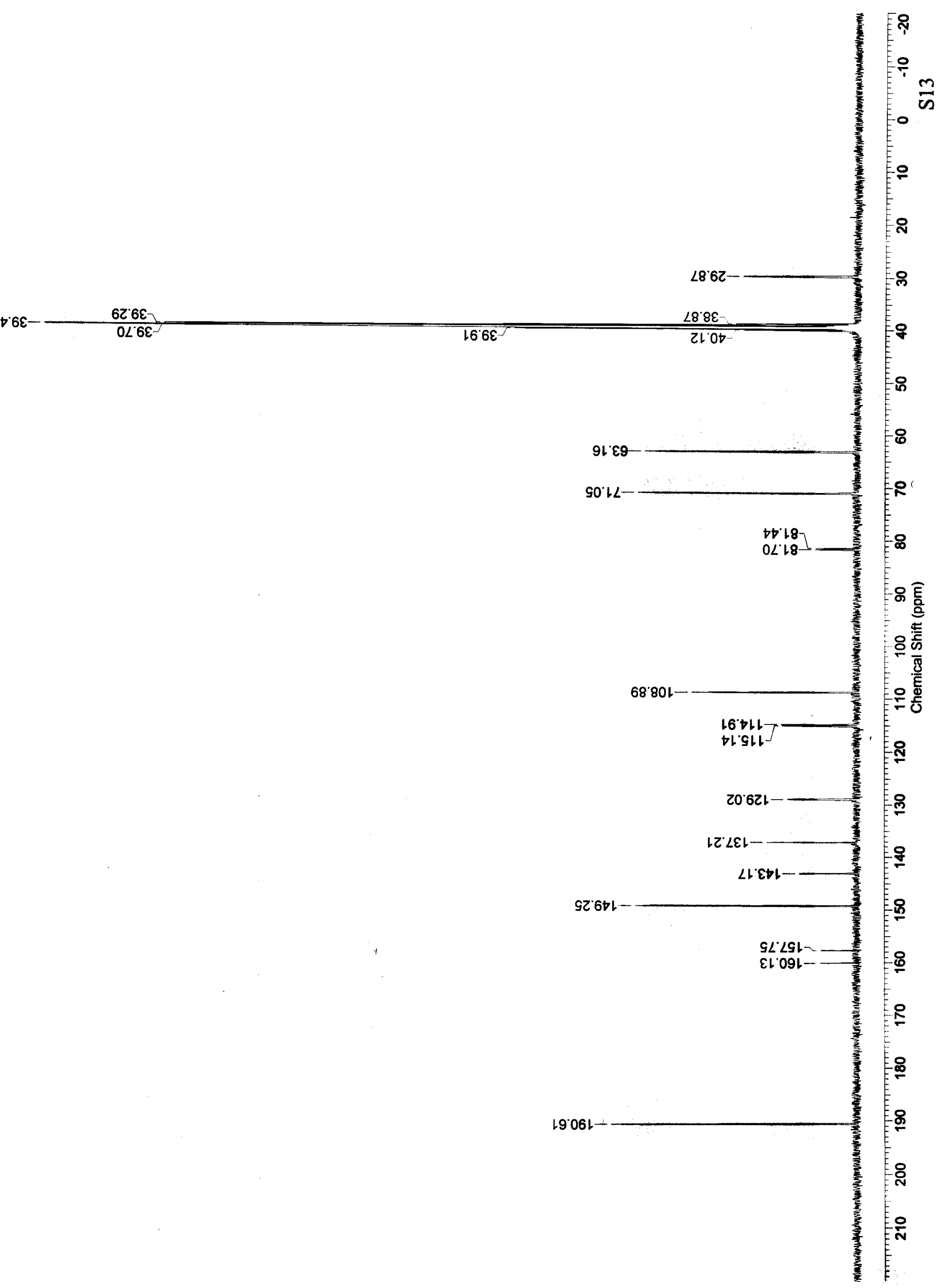

